\title{
Stability Analysis of Nonlinear Time Varying System of Beam-moving Mass Considering Friction Interaction
}

\author{
M. Ghomeshi Bozorg* and M. Keshmiri \\ Department of Mechanical Engineering, Isfahan University of Technology, Isfahan, Iran; \\ m.ghomeshibozorg@me.iut.ac.ir, mehdik@cc.iut.ac.ir
}

\begin{abstract}
In this paper, a new analysis is performed on dynamic behavior of beam-moving mass system, considering all the linear and nonlinear inertia terms of the moving mass as well as the friction between the beam and the mass. The partial deferential governing equation is transferred to a discretized form, using Galerkin method. Then the Homotopy perturbation method is used to solve the nonlinear time varying discretized equation of motion. In addition to the approximate analytic solution of the equation, the border line of stable and unstable regions and the resonance curves in the mass-velocity parametric plane are determined semi-analytically. The numerical simulation is used to verify these new finding from the analysis.
\end{abstract}

Keywords: Beam-moving Mass, Homotopy Perturbation Method, Nonlinear Time Varying System.

\section{Introduction}

Analyzing the dynamic interaction of a beam under the passage of a moving mass received the first attention from the apparition of railways in the nineteenth century and has been studied from different aspects by researchers. In recent years, due to economical reasons, there has been a growing trend towards the construction of more slender structures, inducing more pronounced vibrations. This fact emphasizes the importance of studying the formation of such phenomena and how to control them. Therefore, determining the verge of instability or conditions for appearance of resonance in structures supporting massive traffic is essential; as such instances may result in irreversible dangerous situations.

The research on this subject, neglected inertia terms of the vehicle and considered it as a moving load [1-8]. In many of these cases, vehicle transition recurrence equal to the beam natural frequencies are presented as trigger to resonance. In some other investigations researchers considered only the linear inertia term of the moving mass [9-11]. Only a restricted number of them included all components of moving mass acceleration in their analysis [12-14].

Homotopy methods are among those which have been recently employed to analyze vibrating systems. The extensive works based on homotopy method can be categorized in two groups. The first belongs to the class of convergence techniques for enhancing solutions accuracy which is irrelevant here. The second part focuses on the potential of homotopy methods to solve diverse differential equations. Some of those works which lean on this method for solving dynamic systems are enumerated in references [15-24].

Although there has been a general trend to employ homotopy method in studying and analyzing system's dynamics, nonetheless to the best of knowledge, dynamic stability investigation of a nonlinear time varying system via this approach has not been done yet.

In this paper, for the first time the dynamic behavior of the beam-moving mass system considering all components of the moving mass acceleration as well as the friction between the beam and moving mass, as a nonlinear time variant system, is investigated via homotopy

${ }^{*}$ Corresponding author:

M. Ghomeshi Bozorg (m.ghomeshibozorg@me.iut.ac.ir) 
perturbation method. Results show the performance of this semi-analytical method to predict the limits of instability and resonance. A comparison of the results is performed with numerical solutions, to verify the method accuracy.

\section{Dynamic Modelling of the System}

Figure 1 shows a simply supported uniform EulerBernoulli beam with mass per unit length $m$, length $l$ and bending stiffness $E I$ which is under the influence of a moving mass $M$ with velocity $v$.

The friction coefficient between the moving mass and the beam surface is considered equal to the value $\mu$.

It is assumed that the moving mass always keeps its contact with the beam. The friction force is assumed to be evaluated at every instant by $F_{f}=\mu N$, where, $N$ is the vertical surface reaction at the contact point. This force can be calculated by considering all acceleration components of the moving mass as follows:

$$
N=M\left(g-\frac{\partial^{2} z}{\partial t^{2}}-2 v \frac{\partial^{2} z}{\partial x \partial t}-v^{2} \frac{\partial^{2} z}{\partial x^{2}}\right) \delta(x-v t),
$$

where, $g$ is the gravity intensity and is the delta Dirac function.

The friction force between the moving mass and the beam produces a variable internal axial force in the beam and reaction forces $p_{1}, p_{2}$ at the supports that depending on the direction of motion may be of tensile or compressive nature, as shown in Figure 2.

The static indeterminacy of the problem can be solved by solid mechanics methods, resulting to the following evaluation for the supports axial reactions,

$$
p_{1}=\left(1-\frac{v t}{l}\right) F_{f},
$$

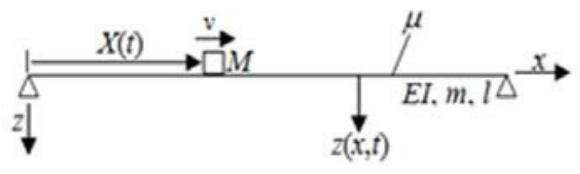

Figure 1. The beam under the effect of a moving mass.

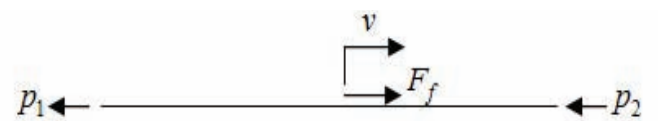

Figure 2. Axial force diagram applied on the beam.

$$
p_{2}=\frac{v t}{l} F_{f}
$$

By adopting the Hamiltonian approach, the partial differential equation governing the beam vibrations under the influence of the moving mass is obtained as follows,

$$
\begin{aligned}
& m \frac{\partial^{2} z}{\partial t^{2}}+\frac{\partial^{2}}{\partial x^{2}}\left(E I \frac{\partial^{2} z}{\partial x^{2}}\right)-\frac{\partial}{\partial x}\left(P \frac{\partial z}{\partial x}\right)=M\left(g-\frac{\partial^{2} z}{\partial t^{2}}-2 v \frac{\partial^{2} z}{\partial x \partial t}\right. \\
& \left.-v^{2} \frac{\partial^{2} z}{\partial x^{2}}\right) \delta(x-v t)
\end{aligned}
$$

where in this relation, $P$ is the axial internal force induced into the beam by the friction force due to the vehicle passage.

\section{Decoupling Governing Equations}

Based on the Galerkin method, the deflection of the beam in Figure 1 is considered as follows,

$$
z(x, t)=\sum_{k=1}^{n} \varphi_{k}(x) q_{k}(t),
$$

where $\varphi_{k}(x)$ is the $k$-th shape function and $q_{k}(t)$ is the corresponding modal coordinate for this function. $n$ is the number of shape functions considered. After substitution, the partial differential equation can be converted to a set of ordinary differential equations on the modal coordinates,

$$
T(t, q) \ddot{q}+Y(t, q) \dot{q}+\Lambda(t, q) q+\Omega(t, q, \dot{q})=f(t),
$$

where, $q$ is the modal coordinates vector and components of the coefficient matrices $T(t), Y(t), \Lambda(t)$ and vectors $\Omega(t, q, \dot{q})$ and $f(t)$ are determined as follows,

$$
\begin{aligned}
& T_{i j}=m \int_{0}^{l} \varphi_{i}(x) \varphi_{j}(x) d x+M \varphi_{j}(v t)\left[\varphi_{i}(v t)-\mu \sum_{k=1}^{n}\left\{\int_{0}^{v t} \varphi_{i}^{\prime}(x)\right.\right. \\
& \left.\left.\varphi_{j}^{\prime}(x) d x-\frac{v t}{l} \int_{0}^{l} \varphi_{i}^{\prime}(x) \varphi_{k}^{\prime}(x) d x\right\} q_{k}(t)\right],
\end{aligned}
$$$$
Y_{i j}=2 M v \varphi_{j}^{\prime}(v t)\left[\varphi_{i}(v t)-\mu \sum_{k=1}^{n}\left\{\int_{0}^{v t} \varphi_{i}^{\prime}(x) \varphi_{k}^{\prime}(x) d x\right.\right.
$$$$
\left.\left.-\frac{v t}{l} \int_{0}^{l} \varphi_{i}^{\prime}(x) \varphi_{k}^{\prime}(x) d x\right\} q_{k}(t)\right] \text {, }
$$ 


$$
\begin{aligned}
& \Lambda_{i j}=E I \int_{0}^{l} \varphi_{i}^{\prime \prime}(x) \varphi_{j}^{\prime \prime}(x) d x+\mu M g\left\{\int_{0}^{v t} \varphi_{i}^{\prime}(x) \varphi_{j}^{\prime}(x) d x\right. \\
& \left.-\frac{v t}{l} \int_{0}^{l} \varphi_{i}^{\prime}(x) \varphi_{j}^{\prime}(x) d x\right\}+M v^{2} \varphi_{j}^{\prime \prime}(v t)\left[\varphi_{i}(v t)-\mu \sum_{k=1}^{n}\left\{\int_{0}^{v t} \varphi_{i}^{\prime}(x)\right.\right. \\
& \left.\left.\varphi_{k}^{\prime}(x) d x-\frac{v t}{l} \int_{0}^{l} \varphi_{i}^{\prime}(x) \varphi_{j}^{\prime}(x) d x\right\} q_{k}(t)\right], \\
& \Omega_{i}=0.5 \mu M v^{2} \sum_{k=1}^{n} \sum_{m=1}^{n}\left[\left\{\int_{0}^{v t} \varphi_{k}^{\prime}(x) \varphi_{m}^{\prime}(x) d x-\frac{v t}{l} \int_{0}^{l} \varphi_{k}^{\prime}(x)\right.\right. \\
& \left.\left.\varphi_{m}^{\prime}(x) d x\right\} q_{k}(t) q_{m}(t)\right] \varphi^{\prime \prime}{ }_{i}(v t) \\
& -\mu M v \sum_{k=1}^{n} \sum_{m=1}^{n}\left[\left\{\left(\frac{v}{l}\right) \int_{0}^{l} \varphi_{k}^{\prime}(x) \varphi_{m}^{\prime}(x) d x-v \varphi_{k}^{\prime}(v t) \varphi_{m}^{\prime}(v t)\right\} q_{k}(t) q_{m}(t)\right. \\
& \left.-\left\{\int_{0}^{v t} \varphi_{k}^{\prime}(x) \varphi_{m}^{\prime}(x) d x-\frac{v t}{l} \int_{0}^{l} \varphi_{k}^{\prime}(x) \varphi_{m}^{\prime}(x) d x\right\}\left(\dot{q}_{k} q_{m}+q_{k} \dot{q}_{m}\right)\right] \varphi_{i}^{\prime}(v t), \\
& f_{i}=M g \varphi_{i}(v t) .
\end{aligned}
$$

By defining normalized shape functions as $\int_{0}^{l} \varphi_{i}(x)$ $\varphi_{i}(x) d x=1$ and selecting only one shape mode $\varphi(x)=\sqrt{2 / l}$ $\sin (\pi x / l)$, the equation governing the corresponding modal coordinate becomes,

$\left(1+\alpha\left(1-\cos 2 \bar{\omega}_{1} t\right)\right) \ddot{q}+\left(2 \alpha \bar{\omega}_{1} \sin 2 \bar{\omega}_{1} t\right) \dot{q}+\left(\omega_{1}^{2}-\alpha \bar{\omega}_{1}^{2}\right.$

$\left.\left(1-\cos 2 \bar{\omega}_{1} t\right)+c_{1} \sin 2 \bar{\omega}_{1} t\right) q$

$-c_{2}\left(\cos \bar{\omega}_{1} t-\cos 3 \bar{\omega}_{1} t\right) q \ddot{q}+c_{3}\left(5 \cos \bar{\omega}_{1} t+3 \cos 3 \bar{\omega}_{1} t\right) q^{2}=$

$\sqrt{2 l} \alpha g \sin \bar{\omega}_{1} t$,

where, $\omega_{1}$ is the first natural frequency of the beam and $\bar{\omega}_{1}$, the non-dimensional parameters $\alpha$ and $\gamma$, and the coefficients $c_{1}, c_{2}, c_{3}$ are defined as follows,

$$
\begin{aligned}
& \alpha=M / m l, \quad \gamma=\pi v / l \omega_{1}, \quad \bar{\omega}_{1}=\gamma \omega_{1} \\
& c_{1}=\pi \mu \alpha g / 2 l, \quad c_{2}=\pi \mu \alpha / \sqrt{8 l^{3}}, \quad c_{3}=\pi \mu \alpha \bar{\omega}_{1}^{2} /\left(\sqrt{32 l^{3}}\right) \text {. }
\end{aligned}
$$

As it can be seen, Eq. (8) is a nonlinear time varying equation which can be solved for any prescribed initial conditions and vehicles parameters like mass and speed. Then by substitution modal coordinate in Eq. (5), the beam response to the moving mass can be calculated at arbitrary locations.

Upon exiting the beam span, the moving mass will no more influence the beam and time-varying coefficients of governing Eq. (8) will vanish, eliminating the conditions for vibrations amplification. If this motion is subsequently followed by the passage of other vehicles for a sufficient lapse of time, the excitation may lead to instability issues.
In order to investigate such conditions, throughout this article it is considered that another similar vehicle enters the beam following the exiting of the precedent. Consequently, the coefficients of Eq. (8) becomes periodical with period $T_{p}=l / v$, about which a Fourier expansion of the coefficients can be performed,

$$
\begin{aligned}
& \left(1+\alpha\left(1-\cos 2 \bar{\omega}_{1} t\right)\right) \ddot{q}+\left(2 \alpha \bar{\omega}_{1} \sin 2 \bar{\omega}_{1} t\right) \dot{q} \\
& +\left(\omega_{1}^{2}-\alpha \bar{\omega}_{1}^{2}\left(1-\cos 2 \bar{\omega}_{1} t\right)+c_{1} \sin 2 \bar{\omega}_{1} t\right) q \\
& -c_{2}\left(\frac{8}{3 \pi}-\sum_{n=1}^{\infty} \frac{4 \cos n \pi}{\pi}\left(\frac{1}{-1+4 n^{2}}+\frac{3}{-9+4 n^{2}}\right) \cos 2 n \bar{\omega}_{1} t\right) q \ddot{q} \\
& +c_{3}\left(\frac{8}{\pi}+\sum_{n=1}^{\infty} \frac{4 \cos n \pi}{\pi}\left(\frac{-5}{-1+4 n^{2}}+\frac{9}{-9+4 n^{2}}\right) \cos 2 n \bar{\omega}_{1} t\right) q^{2} \\
& =\frac{\sqrt{8 l} \alpha g}{\pi}\left(1+\sum_{n=1}^{\infty} \frac{2}{1-4 n^{2}} \cos 2 n \bar{\omega}_{1} t\right) .
\end{aligned}
$$

\section{Stability Analysis by Using Homotopy Perturbation Method}

\subsection{Homotopy Perturbation Method Structure}

The homotopy perturbation method is considered as a perturbation approach to obtain a semi-analytical solution for differential equations by transforming the problem to a set of elementary problems. The most basic characteristic of this method in comparison to other perturbation methods consists on not relying on a small parameter existing in the equation and thus this characteristic permits to solve a more vast range of equations without such restriction.

In order to perform stability analysis for the considered problem, the homotopy perturbation method for solving differential equations presented in [24] is used.

A brief description of how the method applies on differential equation (10) is presented here. Let us rearrange the equation in the following form.

$$
\ddot{q}+R(q, \dot{q}, \ddot{q}, t)=0, \quad q(0)=0, \dot{q}(0)=0,
$$

where, $R$ is a general nonlinear differential operator which easily matched with Eq. (10). In the next step, the original equation is rewritten as

$$
\ddot{q}+1 . q=q-R(q, \dot{q}, \ddot{q}, t),
$$

this is transformed to the requested homotopy form,

$$
\ddot{q}+1 . q=p .(q-R(q, \dot{q}, \ddot{q}, t)),
$$


where, $p \in[0,1]$ is the embedding parameter. For $p=0$, Eq. (13) reduces to a linear differential equation and the main Eq. (11) is reconstituted for $p=1$.

Let us substitute the solution $q$ and the coefficient 1 multiplying $q$ in the Eq. (13) by indeterminate power series of $p$,

$$
\begin{gathered}
q=\sum_{i=0}^{n} p^{i} A_{i}, \\
1=\omega^{2}-\sum_{i=1}^{n} p^{i} \lambda_{i}
\end{gathered}
$$

where, in these relations $A_{i}$ is the solution of the $i$-th order homotopy equation, $\omega$ is the response frequency and $\lambda_{i}$ is the $i$-th coefficient of the expansion. By back substituting the expressions (14) and (15) into the homotopy equation (13), then identifying coefficients of the equal powers results in a set of linear differential equations which have to be solved consecutively. In this trend, secular terms are extracted from the equations and eliminated by setting appropriate values for the coefficients $\lambda_{i}$. Each function $A_{i}$ is determined separately and it is replaced in the next equation. Upon substituting these results into (14) and (15), it suffices to replace $p=1$ to obtain the expanded series solution to the main equation (11) and the vibration frequency.

\subsection{Stability Analysis via Homotopy Solution}

Based on the aforementioned homotopy perturbation method, the homotopy transformation on Eq. (10) leads to following form,

$\ddot{q}+1 . q=p\left[q-\alpha\left(1-\cos 2 \bar{\omega}_{1} t\right) \ddot{q}-\left(2 \alpha \bar{\omega}_{1} \sin 2 \bar{\omega}_{1} t\right) \dot{q}\right.$

$$
-\left(\omega_{1}^{2}-\alpha \bar{\omega}_{1}^{2}\left(1-\cos 2 \bar{\omega}_{1} t\right)+c_{1} \sin 2 \bar{\omega}_{1} t\right) q
$$$$
+c_{2}\left(\frac{8}{3 \pi}-\sum_{n=1}^{\infty} \frac{4 \cos n \pi}{\pi}\left(\frac{1}{-1+4 n^{2}}+\frac{3}{-9+4 n^{2}}\right) \cos 2 n \bar{\omega}_{1} t\right) q \ddot{q}
$$$$
-c_{3}\left(\frac{8}{\pi}+\sum_{n=1}^{\infty} \frac{4 \cos n \pi}{\pi}\left(\frac{-5}{-1+4 n^{2}}+\frac{9}{-9+4 n^{2}}\right) \cos 2 n \bar{\omega}_{1} t\right) q^{2}
$$$$
\left.+\frac{\sqrt{8 l} \alpha g}{\pi}\left(1+\sum_{n=1}^{\infty} \frac{2}{1-4 n^{2}} \cos 2 n \bar{\omega}_{1} t\right)\right] \text {. }
$$

Substituting expansions (14) and (15) instead for $q$ and 1 in Eq. (16), resulting to

$$
\begin{aligned}
& \left(\ddot{A}_{0}+p \ddot{A}_{1}+p^{2} \ddot{A}_{2}+\ldots\right)+\left(\omega^{2}-p \gamma_{1}-p^{2} \gamma_{2}-\ldots\right) \\
& \left(A_{0}+p A_{1}+p^{2} A_{2}+\ldots\right)=p\left[\left(A_{0}+p A_{1}+p^{2} A_{2}+\ldots\right)\right.
\end{aligned}
$$

$$
\begin{aligned}
& -\alpha\left(1-\cos 2 \bar{\omega}_{1} t\right)\left(\ddot{A}_{0}+p \ddot{A}_{1}+p^{2} \ddot{A}_{2}+\ldots\right) \\
& -\left(2 \alpha \bar{\omega}_{1} \sin 2 \bar{\omega}_{1} t\right)\left(\dot{A}_{0}+p \dot{A}_{1}+p^{2} \dot{A}_{2}+\ldots\right) \\
& -\left(\omega_{1}^{2}-\alpha \bar{\omega}_{1}^{2}\left(1-\cos 2 \bar{\omega}_{1} t\right)+c_{1} \sin 2 \bar{\omega}_{1} t\right) \\
& \left(A_{0}+p A_{1}+p^{2} A_{2}+\ldots\right)+c_{2}\left(\frac{8}{3 \pi}-\sum_{n=1}^{\infty} \frac{4 \cos n \pi}{\pi}\left(\frac{1}{-1+4 n^{2}}+\frac{3}{-9+4 n^{2}}\right)\right. \\
& \left.\quad \cos 2 n \bar{\omega}_{1} t\right)\left(A_{0}+p A_{1}+\ldots\right)\left(\ddot{A}_{0}+p \ddot{A}_{1}+\ldots\right) \\
& -c_{3}\left(\frac{8}{\pi}+\sum_{n=1}^{\infty} \frac{4 \cos n \pi}{\pi}\left(\frac{-5}{-1+4 n^{2}}+\frac{9}{-9+4 n^{2}}\right)\right. \\
& \left.\quad \cos 2 n \bar{\omega}_{1} t\right)\left(A_{0}+p A_{1}+p A_{2}+\ldots\right)^{2} \\
& \left.+\frac{\sqrt{8 l} \alpha g}{\pi}\left(1+\sum_{n=1}^{\infty} \frac{2}{1-4 n^{2}} \cos 2 n \bar{\omega}_{1} t\right)\right] .
\end{aligned}
$$

Considering quiescent conditions for the beam prior to the vehicle passage, homotopy equations of order $p^{0}$ and $p^{1}$ in (17) together with initial conditions are determined as follows,

$$
\begin{aligned}
& p^{0}: \ddot{A}_{0}+\omega^{2} A_{0}=0, \quad A_{0}(0)=0, \dot{A}_{0}(0)=0, \quad(18) \\
p^{1}: \ddot{A}_{1} & +\omega^{2} A_{1}=\left(1+\gamma_{1}\right) A_{0}-\alpha\left(1-\cos 2 \bar{\omega}_{1} t\right) \ddot{A}_{0}-\left(2 \alpha \bar{\omega}_{1} \sin 2 \bar{\omega}_{1} t\right) \dot{A}_{0} \\
& -\left(\omega_{1}^{2}-\alpha \bar{\omega}_{1}^{2}\left(1-\cos 2 \bar{\omega}_{1} t\right)+c_{1} \sin 2 \bar{\omega}_{1} t\right) A_{0} \\
+ & c_{2}\left(\frac{8}{3 \pi}-\sum_{n=1}^{\infty} \frac{4 \cos n \pi}{\pi}\left(\frac{1}{-1+4 n^{2}}+\frac{3}{-9+4 n^{2}}\right) \cos 2 n \bar{\omega}_{1} t\right) A_{0} \ddot{A}_{0} \\
& -c_{3}\left(\frac{8}{\pi}+\sum_{n=1}^{\infty} \frac{4 \cos n \pi}{\pi}\left(\frac{-5}{-1+4 n^{2}}+\frac{9}{-9+4 n^{2}}\right) \cos 2 n \bar{\omega}_{1} t\right) A_{0}^{2} \\
+ & \frac{\sqrt{8 l} \alpha g}{\pi}\left(1+\sum_{n=1}^{\infty} \frac{2}{1-4 n^{2}} \cos 2 n \overline{\omega_{1}} t\right), \quad A_{1}(0)=0, \dot{A}_{1}(0)=0 .
\end{aligned}
$$

Solutions to these equations are obtained as follows,

$$
\begin{aligned}
& A_{0}=0, \\
& A_{1}=\frac{\sqrt{8 l} \alpha g}{\pi \omega^{2}}\left(1-\cos \omega t+\sum_{n=1}^{\infty} \frac{2 \omega^{2}\left(\cos 2 n \bar{\omega}_{1} t-\cos \omega t\right)}{\left(1-4 n^{2}\right)\left(\omega^{2}-\left(2 n \bar{\omega}_{1}\right)^{2}\right)} .\right.
\end{aligned}
$$

In order to evaluate $\omega$, the $p^{2}$-order equation extracted from Eq. (17) has to be considered,

$$
\begin{aligned}
& p^{2}: \ddot{A}_{2}+\omega^{2} A_{2}=\gamma_{2} A_{0}+\left(1+\gamma_{1}\right) A_{1}-\alpha\left(1-\cos 2 \bar{\omega}_{1} t\right) \ddot{A}_{1} \\
& -\left(2 \alpha \bar{\omega}_{1} \sin 2 \bar{\omega}_{1} t\right) \dot{A}_{1}-\left(\omega_{1}^{2}-\alpha \bar{\omega}_{1}^{2}\left(1-\cos 2 \bar{\omega}_{1} t\right)+c_{1} \sin 2 \bar{\omega}_{1} t\right) A_{1} \\
& +c_{2}\left(\frac{8}{3 \pi}-\sum_{n=1}^{\infty} \frac{4 \cos n \pi}{\pi}\left(\frac{1}{-1+4 n^{2}}+\frac{3}{-9+4 n^{2}}\right) \cos 2 n \bar{\omega}_{1} t\right) \\
& \left(A_{0} \ddot{A}_{1}+\ddot{A}_{0} A_{1}\right) \\
& -c_{3}\left(\frac{8}{\pi}+\sum_{n=1}^{\infty} \frac{4 \cos n \pi}{\pi}\left(\frac{-5}{-1+4 n^{2}}+\frac{9}{-9+4 n^{2}}\right) \cos 2 n \bar{\omega}_{1} t\right) \\
& \left(2 A_{0} A_{1}\right), A_{2}(0)=0, \dot{A}_{2}(0)=0 .
\end{aligned}
$$


Upon substituting (20) and (21) into (22), a secular term appears which has to be cancelled by equating its coefficient to zero. This leads to the following equation,

$$
1+\lambda_{1}-\omega_{1}^{2}\left(1-\alpha \gamma^{2}\right)+\alpha \omega^{2}=0,
$$

and consequently resulting in the following value for $\lambda_{1}$,

$$
\lambda_{1}=-1+\omega_{1}^{2}\left(1-\alpha \gamma^{2}\right)-\alpha \omega^{2} .
$$

From substituting (24) into (15) and considering $p=1$ in the result, $\omega$ is obtained as follows,

$$
\omega=\omega_{1} \sqrt{\frac{1-\alpha \gamma^{2}}{1+\alpha}} .
$$

Upon eliminating the secular term in Eq. (22), the solution for $A_{2}$ is obtained,

$$
\begin{aligned}
& A_{2}=K_{11} \cos \omega t+K_{12} \sin \omega t+\frac{K_{1}}{\omega^{2}}+\sum_{n=1}^{\infty} \frac{K_{2 n}}{\omega^{2}-\left(2 n \bar{\omega}_{1}\right)^{2}} \cos 2 n \bar{\omega}_{1} t \\
& -\frac{K_{3}}{\omega^{2}-\left(2 \bar{\omega}_{1}+\omega\right)^{2}} \times \cos \left(2 \bar{\omega}_{1}+\omega\right) t-\frac{K_{4}}{\omega^{2}-\left(2 \bar{\omega}_{1}-\omega\right)^{2}} \\
& \cos \left(2 \bar{\omega}_{1}-\omega\right) t-\frac{K_{5}}{\omega^{2}-\left(2 \bar{\omega}_{1}\right)^{2}} \cos 2 \bar{\omega}_{1} t-\sum_{n=1}^{\infty} \frac{K_{6 n}}{\omega^{2}-\left(2 \bar{\omega}_{1}(1+n)\right)^{2}} \\
& \cos 2 \bar{\omega}_{1}(1+n) t-\sum_{n=1}^{\infty} \frac{K_{7 n}}{\omega^{2}-\left(2 \bar{\omega}_{1}(1-n)\right)^{2}} \cos 2 \bar{\omega}_{1}(1-n) t \\
& +K_{8}\left(\frac{\sin \left(2 \bar{\omega}_{1}+\omega\right) t}{\omega^{2}-\left(2 \bar{\omega}_{1}+\omega\right)^{2}}+\frac{\sin \left(2 \bar{\omega}_{1}-\omega\right) t}{\omega^{2}-\left(2 \bar{\omega}_{1}-\omega\right)^{2}}\right)+\frac{K_{9}}{\omega^{2}-\left(2 \bar{\omega}_{1}\right)^{2}} \\
& \sin \left(2 \bar{\omega}_{1} t\right)+\sum_{n=1}^{\infty} K_{10 n}\left(\frac{\sin 2 \bar{\omega}_{1}(1+n) t}{\omega^{2}-\left(2 \bar{\omega}_{1}(1+n)\right)^{2}}+\frac{\sin 2 \bar{\omega}_{1}(1-n) t}{\omega^{2}-\left(2 \bar{\omega}_{1}(1-n)\right)^{2}}\right)
\end{aligned}
$$

which, the coefficients are introduced in the Appendix A.

In case that the expression under the square root in Eq. (25) becomes negative, terms like sinh $\omega t$ and $\cosh \omega t$ appear in the solution leading to instability in the solution. Thus circumstances leading to this condition can be presented as instability criterion. Hence the boundary curve separating regions of stability and instability in $\alpha-\gamma$ plane is indeed the locus of points where $\omega=0$,

$$
\gamma=\sqrt{\frac{1}{\alpha}}
$$

To continue the discussions on the stability analysis, the $p^{3}$-order homotopy equation from (17) has to be considered,

$$
\begin{aligned}
& p^{3}: \ddot{A}_{3}+\omega^{2} A_{3}=\gamma_{3} A_{0}+\gamma_{2} A_{1}+\left(1+\gamma_{1}\right) A_{2} \\
& -\alpha\left(1-\cos 2 \bar{\omega}_{1} t\right) \ddot{A}_{2}-\left(2 \alpha \bar{\omega}_{1} \sin 2 \bar{\omega}_{1} t\right) \dot{A}_{2}
\end{aligned}
$$

$$
\begin{aligned}
& -\left(\omega_{1}^{2}-\alpha \bar{\omega}_{1}^{2}\left(1-\cos 2 \bar{\omega}_{1} t\right)+c_{1} \sin 2 \bar{\omega}_{1} t\right) A_{2} \\
& +c_{2}\left(\frac{8}{3 \pi}-\sum_{n=1}^{\infty} \frac{4 \cos n \pi}{\pi}\left(\frac{1}{-1+4 n^{2}}+\frac{3}{-9+4 n^{2}}\right)\right. \\
& \left.\cos 2 n \bar{\omega}_{1} t\right)\left(A_{0} \ddot{A}_{2}+A_{1} \ddot{A}_{1}+A_{2} \ddot{A}_{0}\right) \\
& -c_{3}\left(\frac{8}{\pi}+\sum_{n=1}^{\infty} \frac{4 \cos n \pi}{\pi}\left(\frac{-5}{-1+4 n^{2}}+\frac{9}{-9+4 n^{2}}\right)\right. \\
& \left.\cos 2 n \bar{\omega}_{1} t\right)\left(2 A_{0} A_{2}+A_{1}^{2}\right), A_{3}(0)=0, \dot{A}_{3}(0)=0 .
\end{aligned}
$$

By substituting $A_{0}, A_{1}, A_{2}$ respectively from (20), (21) and (26) into (28), the coefficient of the secular term has to be cancelled which results in the following equation for $\lambda_{2}$

$$
\lambda_{2}=\frac{\alpha^{2}}{8}\left(3 \omega^{2}+\bar{\omega}_{1}^{2}+\frac{64 g \mu}{3 \pi l}\right)
$$

By back inserting the quantities for $\lambda_{1}$ and $\lambda_{2}$ respectively from (24) and (29) into (15), with inserting $p=1$ in the result, the value for vibration frequency $\omega$ can be calculated as follows,

$$
\omega=\omega_{1} \sqrt{\frac{1-\alpha \gamma^{2}+\frac{1}{8} \alpha^{2} \gamma^{2}+\frac{8 \mu \alpha^{2} g}{3 \pi l \omega_{1}^{2}}}{1+\alpha-\frac{3}{8} \alpha^{2}}} .
$$

In the realistic interval of parameters $0<\alpha<1$ and $0<\gamma<4$, the conditions for negative value under the square root of expression (30) can lead to instability conditions. Therefore, the boundary between stability and instability regions, the locus of points where $\omega=0$ is obtained as follows,

$$
\gamma=\sqrt{\frac{1+\frac{8 \mu \alpha^{2} g}{3 \pi l \omega_{1}^{2}}}{\alpha-\frac{\alpha^{2}}{8}}},
$$

where, the influence of the friction coefficient is evident on the stability boundary relation. This influence is clearly depended on the value of $8 \alpha^{2} g / 3 \pi l \omega_{1}^{2}$.

The stability boundaries obtained up to the $p^{2}$ order and $p^{3}$-order, Eqs. (27) and (31) respectively, are compared in Figure 3 with quantities $\mu=0,0.5,1$ for the friction coefficient and the parameters $E I=500000 \mathrm{~N} \cdot \mathrm{m}^{2}, l=20 \mathrm{~m}, \mathrm{~m}=10 \mathrm{~kg} / \mathrm{m}$ for the beam,

Note that due to the small value of $8 \alpha^{2} g / 3 \pi l \omega_{1}^{2}$ for the current problem, increasing the friction coefficient doesn't much influence the boundary curve and results obtained from different expansion orders are very close. To confirm 
these results, the time responses at the midpoint of the beam span have been obtained under the effect of moving masses with characteristics $(\alpha=0.3, \gamma=1)$ and $(\alpha=0.3, \gamma=2.5)$ located in the stable and unstable zone respectively with friction coefficient $\mu=0.5$. These responses are shown in Figure 4 and 5.

As it can be seen, these numerical simulation results confirm the presented stability analysis.

\section{Resonance Conditions}

After providing the necessary conditions for eliminating the secular terms in (28) and (22), the analysis can be fur-

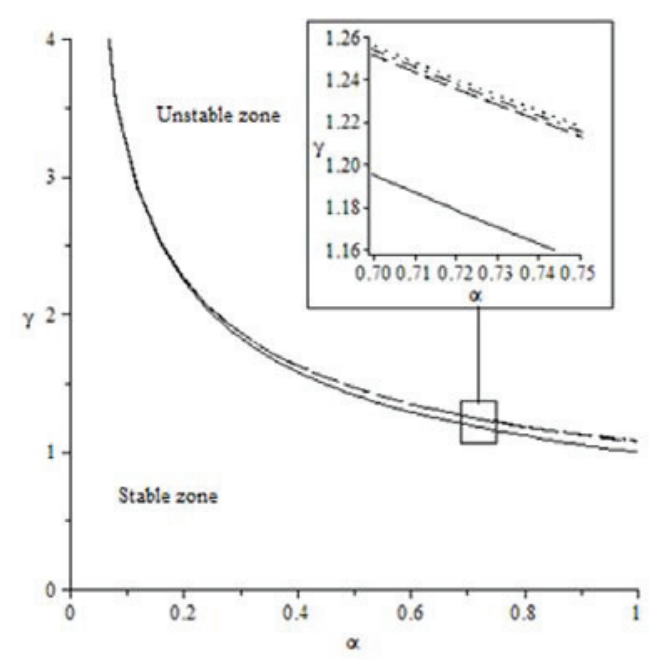

Figure 3. Stability boundary for $\mu=0$ (dashed line), $\mu=0.5$ (dotted-dashed line), $\mu=1$ (dotted line) and the same curve obtained to $p^{2}$-order (solid line).

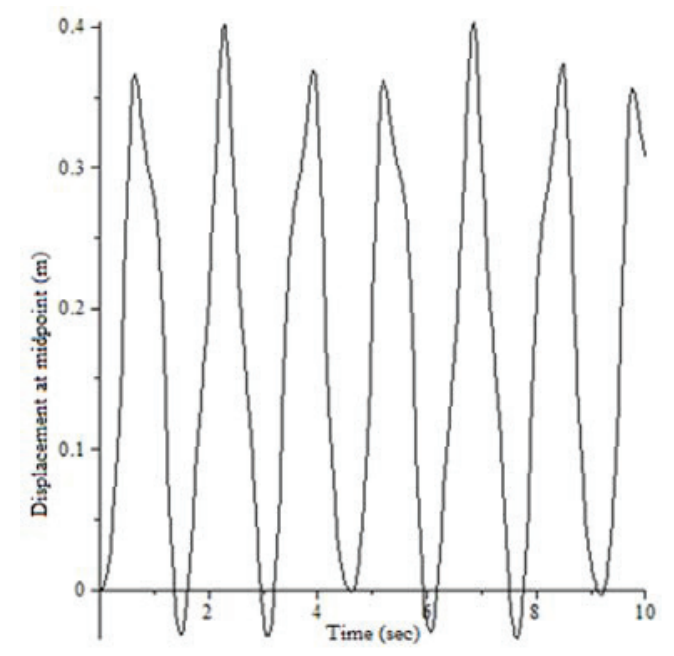

Figure 4. Response to moving mass with characteristics $\gamma=1, \alpha=0.3$ and $\mu=0.5$ lying in the stable zone. ther continued to determine the conditions of resonance in the stable region. Such conditions can be identified by analyzing when the harmonic forcing functions present in the right side of the equations beat with a rhythm equal to the natural frequencies of the equations.

These resonant frequencies are given by

$$
\omega=2 n \bar{\omega}_{1}, \quad n=1,2,3, \ldots
$$

Substituting the resulted term for $\omega$ from (30) into (33), based on the $p^{3}$-order homotopy expansion, the locus of points in the $\alpha-\gamma$ plane where resonance is expected, is obtained in the following term

$$
\gamma=\sqrt{\frac{1+\frac{8 \mu \alpha^{2} g}{3 \pi l \omega_{1}^{2}}}{4 n^{2}\left(1+\alpha-\frac{3}{8} \alpha^{2}\right)+\left(\alpha-\frac{\alpha^{2}}{8}\right)}}, \quad n=1,2,3, \ldots
$$

The locus of points in the $\alpha-\gamma$ plane indicated by Eq. (34) for $n=1,2,3$ and $\mu=0,0.5,1$ is shown in Figure 6 .

Similar to the boundary curves of stability for the current problem, increasing the friction coefficient has a little effect on the curves. In order to evaluate the accuracy of the analysis, the beam midpoint deflection is again numerically obtained corresponding to two sets of $\alpha$ and $\gamma,(\alpha=0.4, \gamma=0.21419)$ and $(\alpha=0.4, \gamma=0.26419)$. The first one is located on the evaluated resonance curve $n=2$ with, $\mu=0.5$ while the second one is in its vicinity. These numerical simulations are shown respectively in Figures 7 and 8.

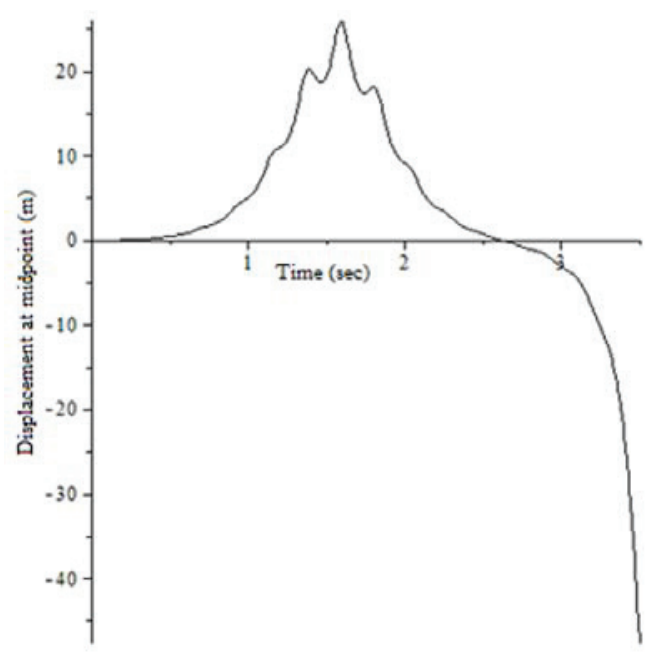

Figure 5. Response to moving mass with characteristics $\gamma=2.5, \alpha=0.3$ and $\mu=0.5$ lying in the unstable zone. 
As expected, because of the approximated nature of the results obtained by $p^{3}$-order expansion, the system's behavior corresponding to the point set on the resonant curve presents beating which shows the vicinity of this point to the real resonance condition. The set of stability boundary and resonance curves are shown in Figure 9 for the value $\mu=0.5$.

\section{Investigation of the Effect of Friction on the Beam's Dynamic Response}

Figure 10 shows time responses at the beam midpoint for two cases $\mu=0$ and $\mu=1$ under the effect of moving

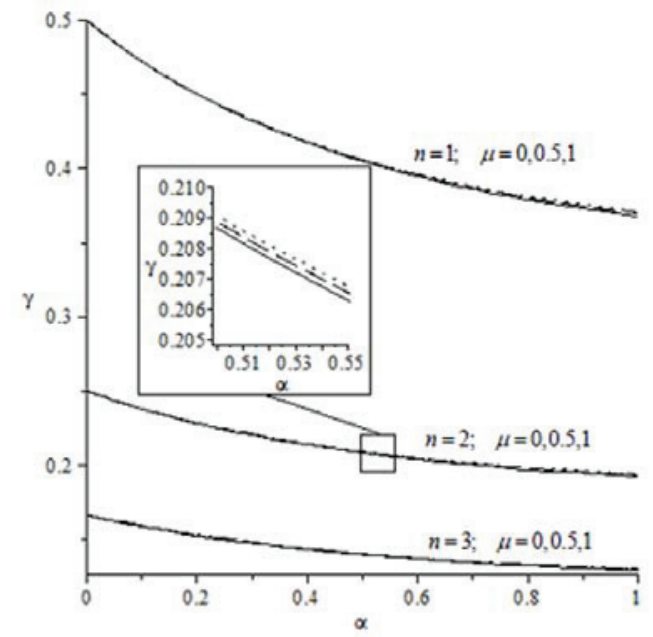

Figure 6. Locus of resonant conditions for $n=1,2,3$ and $\mu=0$ (solid line), $\mu=0.5$ (dashed line), $\mu=1$ (dotted line), up to $p^{3}$ order.

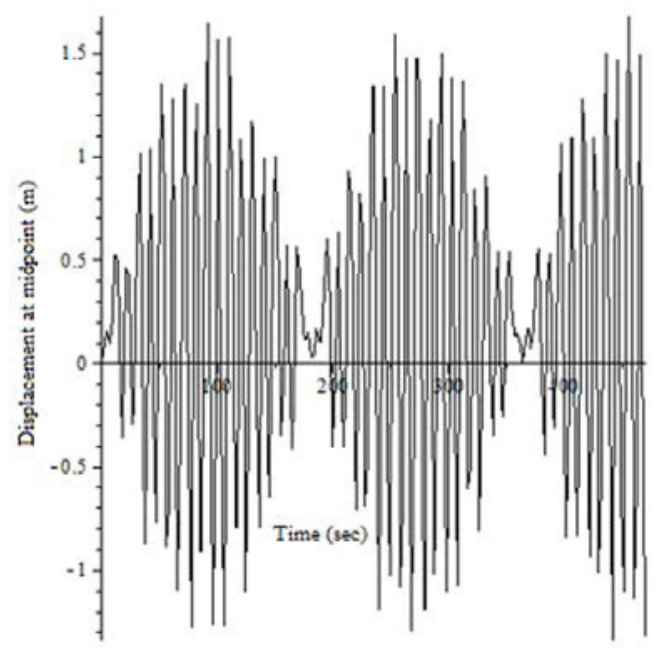

Figure 7. Response to moving mass with characteristics $\gamma=0.21419, \alpha=0.4, \mu=0.5$ lying on the $n=2$ resonance curve. masses with characteristics $(\alpha=0.25, \gamma=1)$. Results show that friction in the current system has negligible effect on the dynamic response.

\section{Conclusions}

A new analysis on the dynamics and stability of beammoving mass system was performed considering all the inertia terms of the moving mass as well as the friction between the beam and the moving mass. Results show that the dynamic model equation of this system is indeed nonlinear time varying. The dynamic stability of this system

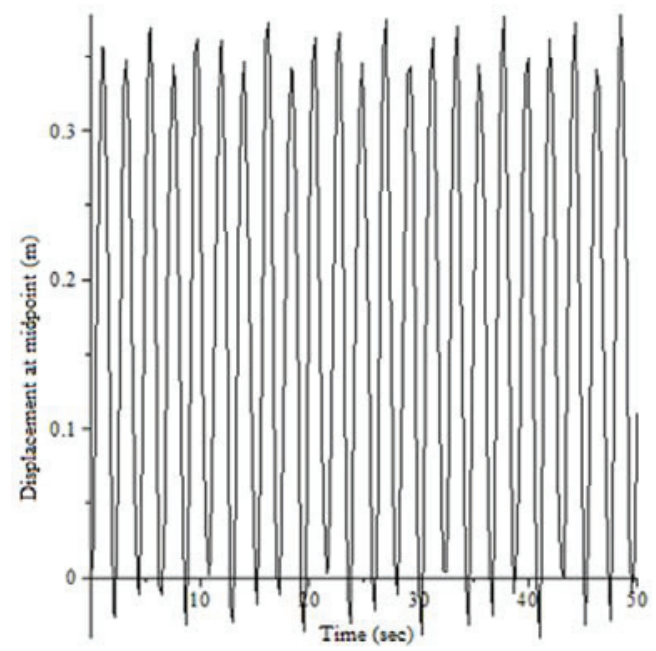

Figure 8. Response to moving mass with characteristics $\gamma=0.26419, \alpha=0.4, \mu=0.5$ lying in the vicinity of the point selected in Figure 7.

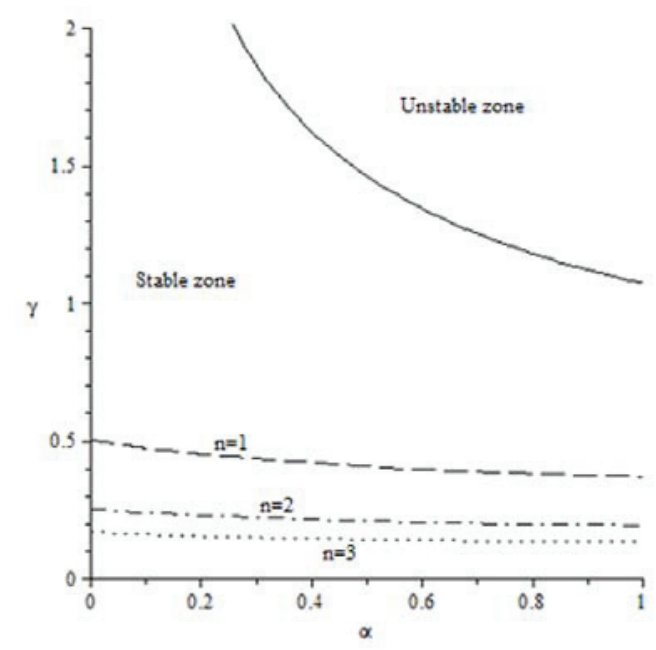

Figure 9. Whole set of stability boundary and resonance curves obtained to the $p^{3}$-order considering friction coefficient $\mu=0.5$. 


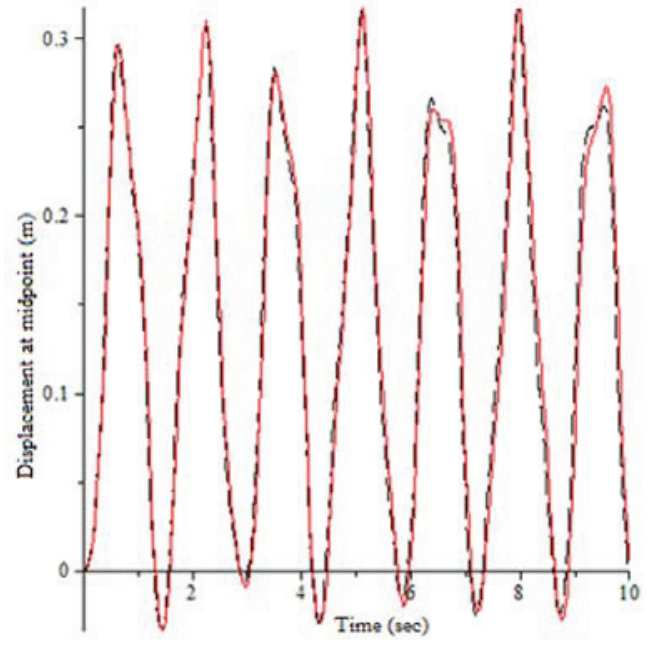

Figure 10. Response to moving mass with characteristics $\alpha=0.25, \gamma=1$ for $\mu=1$ (dashed line) and $\mu=0$ (solid line).

was investigated by the homotopy perturbation method permitting to split the mass-speed parameter plane in stable and unstable regions and determine the border line semi-analytically. The finding of the analysis is completed by specifying the locus of resonant points in the parametric plane. Furthermore, it was shown that friction between the moving mass and beam affects the stability margins and resonance curves through the term of $8 a^{2} g / 3 \pi l \omega_{1}^{2}$.

\section{References}

1. Yau J D, and Fryba L (2007). Responses of suspended beam due to moving loads and vertical seismic ground excitation, Engineering Structures, vol 29(12), 3255-3262.

2. Yau J D (2009). Dynamic response analysis of suspended beams subjected to moving vehicles and multiple support excitations, Journal of Sound and Vibration, vol 325(4-5), 907-922.

3. Garinei A, and Risitano G (2008). Vibration of railway bridges for high speed trains under moving loads varying in time, Engineering Structures, vol 30(3), 724-732.

4. Ju S H, Lin H T et al. (2009). Dominant frequencies of train-induced vibrations, Journal of Sound and Vibration, vol 319(1-2), 247-259.

5. Yang Y B, Wu C M et al. (2001). Dynamic responses of a horizontally curved beam subjected to vertical and horizontal moving loads, Journal of Sound and Vibration, vol 242(3), 519-537.

6. Yang Y B, Lin C L et al. (2004). Mechanism of resonance and cancellation for train-induced vibrations on bridges with elastic bearings, Journal of Sound and Vibration, vol 269(1-2), 345-360.
7. Yau J D, and Yang Y B (2006). Vertical accelerations of simple beams due to successive loads traveling at resonant speeds, Journal of Sound and Vibration, vol 289(1-2), 210-228.

8. Ruzzene M, and Baz A (2006). Dynamic stability of periodic shells with moving loads, Journal of Sound and Vibration, vol 296(4-5), 830-844.

9. Verichev S N, and Metrikine A V (2002). Instability of a bogie moving on flexibly supported timoshenko beam, Journal of Sound and Vibration, vol 253(3), 635-668.

10. Verichev S N, and Metrikine A V (2003). Instability of vibrations of mass that moves uniformly along a beam on a periodically inhomogeneous foundation, Journal of Sound and Vibration, vol 260(5), 901-925.

11. Wang J F, Lin C C et al. (2003). Vibration suppression for high-speed railway bridges using tuned mass dampers, International Journal of Solids and Structures, vol 40(2), 465-491.

12. Rao G V (2000). Linear dynamics of an elastic beam under moving loads, Journal of Vibration and Acoustic, vol 122(3), 281-289.

13. Wang Y M (2002). The dynamic analysis of a beam-mass system due to the occurrence of two-component parametric response, Journal of Sound and Vibration, vol 258(5), 951-967.

14. Zheng D Y, and Fan S C (2002). Instability of vibration of a moving-train-and-rail coupling system, Journal of Sound and Vibration, vol 255(2), 243-259.

15. Ahmadian M T, Mojahedi M et al. (2009). Free vibration analysis of a nonlinear beam using homotopy and modified lindstedt-poincare methods, Journal of Solid Mechanics, vol 1, 29-36.

16. Pirbodaghi T, Ahmadian M T et al. (2009). On the homotopy analysis method for non-linear vibration of beams, Mechanics Research Communications, vol 36(2), 143-148.

17. Ganji S S, Ganji D D et al. (2010). Application of AFF and HPM to the systems of strongly nonlinear oscillation, Current Applied Physics, vol 10(5), 1317-1325.

18. Cveticanin L (2009). Application of homotopy-perturbation to non-linear partial differential equations, Chaos, Solitons and Fractals, vol 40(1), 221-228.

19. Temimi H, Ansari A R et al. (2011). An approximate solution for the static beam problem and nonlinear integrodifferential equations, Computers and Mathematics with Applications, vol 62(8), 3132-3139.

20. Wang J, Chen J K et al. (2008). An explicit solution of the large deformation of a cantilever beam under point load at the free tip, Journal of Computational and Applied Mathematics, vol 212(2), 320-330.

21. Hoseini S H, Pirbodaghi T et al. (2009). On the large amplitude free vibrations of tapered beams: an analytical approach, Mechanics Research Communications, vol 36(8), 892-897.

22. Rafiq A, Malik M Y et al. (2010). Solution of nonlinear pull-in behavior in electrostatic micro-actuators by using 
He's homotopy perturbation method, Computers and Mathematics with Applications, vol 59(8), 2723-2733.

23. Pirbodaghi T, Fesanghary $M$ et al. (2011). Non-linear vibration analysis of laminated composite plates resting on nonlinear elastic foundations, Journal of the Franklin Institute, vol 348(2), 353-368.
24. Ozis T, and Yildirim A (2007). A comparative study of He's homotopy perturbation method for determining frequency-amplitude relation of a nonlinear oscillator with discontinuities, International Journal of Nonlinear Sciences and Numerical Simulation, vol 8(2), 243-248. 


\section{Appendix A: Coefficients of the Solution $A_{2}$ presented in (26)}

$$
\begin{aligned}
& K_{1}=-\frac{2 \sqrt{2 l} \alpha^{2} g}{\pi}, \\
& K_{2 n}=-\frac{4 \sqrt{2 l} \alpha^{2} g}{\pi\left(1-4 n^{2}\right)}, \\
& K_{3}=-\frac{\sqrt{2 l} \alpha^{2} g}{\pi}\left(\bar{\omega}_{1}+\omega\right)^{2}\left(\frac{1}{\omega^{2}}+\sum_{n=1}^{\infty} \frac{2}{\left(1-4 n^{2}\right)\left(\omega^{2}-\left(2 n \bar{\omega}_{1}\right)^{2}\right)}\right) \text {, } \\
& K_{4}=-\frac{\sqrt{2 l} \alpha^{2} g}{\pi}\left(\bar{\omega}_{1}-\omega\right)^{2}\left(\frac{1}{\omega^{2}}+\sum_{n=1}^{\infty} \frac{2}{\left(1-4 n^{2}\right)\left(\omega^{2}-\left(2 n \bar{\omega}_{1}\right)^{2}\right)}\right) \text {, } \\
& K_{5}=\frac{2 \sqrt{2 l} g\left(\alpha \bar{\omega}_{1}\right)^{2}}{\pi \omega^{2}} \\
& K_{6 n}=\frac{2 \sqrt{2 l} g\left((2 n+1) \alpha \bar{\omega}_{1}\right)^{2}}{\pi\left(1-4 n^{2}\right)\left(\omega^{2}-\left(2 n \bar{\omega}_{1}\right)^{2}\right)}, \\
& K_{7 n}=\frac{2 \sqrt{2 l} g\left((2 n-1) \alpha \bar{\omega}_{1}\right)^{2}}{\pi\left(1-4 n^{2}\right)\left(\omega^{2}-\left(2 n \bar{\omega}_{1}\right)^{2}\right)} \text {, } \\
& K_{8}=\frac{\mu(\alpha g)^{2}}{\sqrt{2 l}}\left(\frac{1}{\omega^{2}}+\sum_{n=1}^{\infty} \frac{2}{\left(1-4 n^{2}\right)\left(\omega^{2}-\left(2 n \bar{\omega}_{1}\right)^{2}\right)}\right), \\
& K_{9}=-\sqrt{\frac{2}{l}} \frac{\mu(\alpha g)^{2}}{\omega^{2}} \\
& K_{10 n}=-\sqrt{\frac{2}{l}} \frac{\mu(\alpha g)^{2}}{\left(1-4 n^{2}\right)\left(\omega^{2}-\left(2 n \bar{\omega}_{1}\right)^{2}\right)}, \\
& K_{11}=-\frac{K_{1}}{\omega^{2}}-\sum_{n=1}^{\infty} \frac{K_{2 n}}{\omega^{2}-\left(2 n \bar{\omega}_{1}\right)^{2}}+\frac{K_{3}}{\omega^{2}-\left(2 \bar{\omega}_{1}+\omega\right)^{2}}+\frac{K_{4}}{\omega^{2}-\left(2 \bar{\omega}_{1}-\omega\right)^{2}}+\frac{K_{5}}{\omega^{2}-\left(2 \bar{\omega}_{1}\right)^{2}} \\
& +\sum_{n=1}^{\infty} \frac{K_{6 n}}{\omega^{2}-\left(2 \bar{\omega}_{1}(1+n)\right)^{2}}+\sum_{n=1}^{\infty} \frac{K_{7 n}}{\omega^{2}-\left(2 \bar{\omega}_{1}(1-n)\right)^{2}}, \\
& K_{12}=-\frac{1}{\omega}\left(K_{8}\left(\frac{\left(2 \bar{\omega}_{1}+\omega\right)}{\omega^{2}-\left(2 \bar{\omega}_{1}+\omega\right)^{2}}+\frac{\left(2 \bar{\omega}_{1}-\omega\right)}{\omega^{2}-\left(2 \bar{\omega}_{1}-\omega\right)^{2}}\right)+\frac{2 \bar{\omega}_{1} K_{9}}{\omega^{2}-\left(2 \bar{\omega}_{1}\right)^{2}}\right. \\
& \left.+\sum_{n=1}^{\infty} 2 \bar{\omega}_{1} K_{10 n}\left(\frac{1+n}{\omega^{2}-\left(2 \bar{\omega}_{1}(1+n)\right)^{2}}+\frac{1-n}{\omega^{2}-\left(2 \bar{\omega}_{1}(1-n)\right)^{2}}\right)\right) .
\end{aligned}
$$

